\title{
Optimal partial regularity of very weak solutions to nonhomogeneous A-harmonic systems
}

\section{Qing Zhao* and Shuhong Chen}

"Correspondence:

zhaoqing9283@163.com School of Mathematics and

Statistics, Minnan Normal University, Fujian, Zhangzhou 363000, China

\begin{abstract}
We study partial regularity of very weak solutions to some nonhomogeneous A-harmonic systems. To obtain the reverse Hölder inequality of the gradient of a very weak solution, we construct a suitable test function by Hodge decomposition. With the aid of Gehring's lemma, we prove that these very weak solutions are weak solutions. Further, we show that these solutions are in fact optimal Hölder continuity based on A-harmonic approximation technique.
\end{abstract}

Keywords: nonhomogeneous A-harmonic systems; very weak solution; optimal partial regularity; Hodge decomposition; A-harmonic approximation technique

\section{Introduction}

We consider optimal partial regularity of very weak solutions to nonhomogeneous A-harmonic systems of the following type:

$$
-\operatorname{div} A(x, u, D u)=f(x)
$$

where $u: \Omega \rightarrow R^{N}$ is a vector-valued function on a bounded domain $\Omega \subset R^{n}(n \geq 2)$, and $D u=\left\{D_{\alpha} u^{i}\right\}(1 \leq \alpha \leq n, 1 \leq i \leq N)$ stands for the gradient matrix of $u, A(x, u, \xi): \Omega \times$ $R^{N} \times R^{n N} \rightarrow R^{n N}$ is a measurable function, and $A_{i}^{\alpha}(x, u, \xi)(1 \leq \alpha \leq n, 1 \leq i \leq N)$ are of class $C^{1}$ in $\xi$. To define the very weak solutions to systems (1.1) and obtain the optimal partial regularity results, we need to impose certain structural and regularity conditions on $A$ and to restrict $u$ and $f$ to a particular class of functions as follows: for some $p \geq 2$,

(H1) $A$ is a bounded operator, that is, there exists a constant $\beta>0$ such that

$$
|A(x, u, \xi)| \leq \beta\left(1+|\xi|^{2}\right)^{\frac{p-1}{2}} \quad \text { for all }(x, u, \xi) \in \Omega \times R^{N} \times R^{n N} ;
$$

(H2) $A$ is differentiable with respect to $\xi \in R^{n N}$, that is, there exists a constant $\alpha>0$ such that

$$
D_{\xi} A(x, u, \xi) \zeta \cdot \zeta \geq \alpha\left(1+|\xi|^{2}\right)^{\frac{p-2}{2}}|\zeta|^{2}
$$

for all $x \in \Omega, u \in R^{N}$, and $\xi, \zeta \in R^{n N}$;

\section{Springer}


(H3) there exist a constant $\gamma \in(0,1)$ and a nondecreasing function $K:[0, \infty) \rightarrow[0, \infty)$ such that

$$
|A(x, u, \xi)-A(\tilde{x}, \tilde{u}, \xi)| \leq K(|u|)\left(|x-\tilde{x}|^{p}+|u-\tilde{u}|^{p}\right)^{\frac{\gamma}{p}}(1+|\xi|)^{\frac{p}{2}}
$$

for all $x, \tilde{x} \in \Omega, u, \tilde{u} \in R^{N}$ and $\xi \in R^{n N}$; without loss of generality, we take $K \geq 1$;

(H4) $f$ is a given vector field in $R^{N}$ of class $L_{\text {loc }}^{\frac{n q}{n(p-1)+q}}(\Omega), q>p$.

Under these assumptions, we can now define very weak solutions to (1.1).

Definition 1 A mapping $u \in W_{\text {loc }}^{1, r}(\Omega), p-1 \leq r<p$, is called a very weak solution to (1.1) if

$$
\int_{\Omega} A(x, u, D u) \cdot D \phi d x=\int_{\Omega} f(x) \phi d x
$$

for all $\phi \in W_{0}^{1, \frac{r}{r-p+1}}(\Omega)$.

In order to improve the integrability of a very weak solution to (1.1), we need to prove a suitable reverse Hölder inequality. In 1973, Gehring [1] discovered the crucial selfimproving property of the reverse Hölder inequality and applied it to establish higher integrability of $n$-dimensional $k$-quasiconformal mapping. Subsequently, Meyers and Elcrat [2] generalized this inequality based on Caccioppoli's inequality. They improved the integrability of weak solutions to nonlinear elliptic systems with the help of Gehring's lemma. Especially, they pointed out that regularity properties remained valid in a somewhat slightly larger Sobolev space to linear elliptic systems depending on the duality. In fact, this regularity result about very weak solutions was first showed by Meyers [3] in 1963. Unfortunately, neither the method used in [2] for proving the reverse Hölder inequality nor the duality employed in $[2,3]$ can be applied to deal with very weak solutions to nonlinear elliptic systems. To overcome these difficulties, Lewis [4] used the technique of harmonic analysis and successfully proved that very weak solutions to nonlinear elliptic systems are indeed weak solutions. Later Iwaniec and Sbordone [5] achieved a similar result via the methods of Hodge decomposition and prior estimation.

Since then, studies on properties of very weak solutions to partial differential equations, especially for regularity of very weak solutions to A-harmonic systems, have attracted considerable attention. Following the method of Iwaniec and Sbordone [5], Giachetti, Leonetti, and Schiachi [6] obtained the partial regularity result of A-harmonic systems $\operatorname{div} A(x, u, D u)=0$. Tong, $\mathrm{Gu}$, and $\mathrm{Xu}$ [7] extended their result to nonhomogeneous A-harmonic systems $\operatorname{div} A(x, D u)=f(x)$ and improved the integrability of very weak solutions. Greco, Iwaniec, and Sbordone [8] even applied this method to the $p$-harmonic equation $\operatorname{div}|D u|^{p-2} D u=\operatorname{div} f$.

Motivated by these works, we mainly consider the optimal partial regularity to nonhomogeneous A-harmonic systems in the form of (1.1) under assumptions (H1)-(H4).

For the sake of desired results, we first need to improve the exponent of integrability for the gradient of a very weak solution to an even slightly better one than the natural exponent $p$. The crucial difficulty is to construct an appropriate test function below the natural exponent. In this article, we follow the spirit of Iwaniec and Sbordone [5] using the Hodge 
decomposition to construct it. Combining the Sobolev imbedding theorem, Young's inequality, Poincaré's inequality, and so on, we improve the exponents of integrability of very weak solutions to (1.1). In other words, we successfully prove that very weak solutions to (1.1) are in fact weak solutions. More precisely, we obtain the following result.

Theorem 1 Let $u$ be a very weak solution to systems (1.1). Assume that the structure conditions (H1), (H2), and (H4) hold. Then there exist exponents $p-1<r_{1}=r_{1}(n, N, p, \alpha, \beta)<$ $p<r_{2}=r_{2}(n, N, p, \alpha, \beta)<\infty$ such that $u \in W_{\text {loc }}^{1, r_{1}}(\Omega)$ belongs to $W_{\text {loc }}^{1, r_{2}}(\Omega)$.

A direct consequence of this result follows immediately.

Corollary 1 Under the assumptions of Theorem 1 , there exists $r_{1}=r_{1}(n, N, p, \alpha, \beta)<p$ such that every very weak solution $u \in W_{\mathrm{loc}}^{1, r}(\Omega)$ with $r_{1}<r<p$ belongs to $W_{\mathrm{loc}}^{1, p}(\Omega)$.

Further, we establish the optimal partial regularity result of very weak solutions to (1.1). Generally speaking, we cannot expect that weak solutions to (1.1) will be $C^{2}$-solutions even under reasonable assumptions on operator $A$ and $f$. This is initially pointed out by De Giorgi $[9,10]$ and Giusti and Miranda [11]. Thus, our aim is to obtain the optimal Hölder continuity of very weak solutions to (1.1). Fortunately, we achieve it by means of A-harmonic approximation technique and obtain the optimal Hölder continuity $C^{1, \gamma}$ in the regular set of the following:

Theorem 2 Let $u \in W_{\text {loc }}^{1, r}(\Omega), r_{1}<r<p$, be a very weak solution to (1.1). Consider $r_{1}$ as in Corollary 1. Suppose that assumptions (H1)-(H4) hold. Then there exists an open set $\Omega_{0} \subset \Omega$ such that $u \in C^{1, \gamma}\left(\Omega_{0}\right)$ for $\gamma$ is defined in $(\mathrm{H} 3)$. We have

$$
\Omega-\Omega_{0}=\Sigma_{1} \cup \Sigma_{2}
$$

where

$$
\Sigma_{1}=\left\{x_{0} \in \Omega: \liminf _{R \rightarrow 0^{+}} f_{B_{R}\left(x_{0}\right)}\left|D u-(D u)_{x_{0}, R}\right|^{p} d x>0\right\}
$$

and

$$
\Sigma_{2}=\left\{x_{0} \in \Omega: \limsup _{R \rightarrow 0^{+}}\left(\left|u_{x_{0}, R}\right|+\left|(D u)_{x_{0}, R}\right|\right)=\infty\right\} .
$$

Moreover, we have $\left|\Omega-\Omega_{0}\right|=0$.

To close this section, we briefly summarize the notation used in this paper. As noted before, we consider a bounded domain $\Omega \subset R^{n}(n \geq 2)$ and mappings from $\Omega$ to $R^{N}$. We write $B_{r}\left(x_{0}\right)=\left\{x \in \Omega:\left|x-x_{0}\right|<r\right\}, x_{0} \in \Omega$. For a given set $X$, we denote by $|X|$ its $n$-dimensional Lebesgue measure. If $|X|>0$, then the average of a given $g \in L^{1}(X)$ over $X$ is denoted by $f_{X} g d x$, that is, $f_{X} g d x=\frac{1}{|X|} \int_{X} g d x$. In particular, we write $g_{x_{0}, r}=f_{B_{r}\left(x_{0}\right)} g d x$. Let $\alpha_{n}$ denote the volume of the unit ball in $R^{n}$, that is, $\alpha_{n}=\left|B_{1}(0)\right|$, then $\left|B_{r}\left(x_{0}\right)\right|=\alpha_{n} r^{n}$.

The rest of this paper is arranged as follows. In Section 2, we provide some necessary preliminary lemmas. In Section 3, we prove the main results. 


\section{Preliminary lemmas}

Before proving the results, we state a few useful lemmas.

The first one is a stability result of the Hodge decomposition, from which we could construct a suitable test-function concerning estimates below the natural exponent for (1.1).

Lemma 1 ([5]) Let $\Omega \subset R^{n}$ be a regular domain, and $w \in W_{0}^{1, r}\left(\Omega, R^{N}\right), r>1$, and let $-1<\epsilon<r-1$. Then there exist $\phi \in W_{0}^{1, \frac{r}{1+\epsilon}}\left(\Omega, R^{N}\right)$ and a divergence-free matrix field $H \in L^{\frac{r}{1+\epsilon}}\left(\Omega, R^{n N}\right)$ such that

$$
|D w|^{\epsilon} D w=D \phi+H .
$$

Moreover,

$$
\|H\|_{\frac{r}{1+\epsilon}} \leq C_{r}(\Omega, N)|\epsilon|\|D w\|_{r}^{1+\epsilon} .
$$

The most useful case for us in Lemma 1 is where $\epsilon$ is negative. For $u \in W_{\text {loc }}^{1, r}(\Omega)$ with $p-1<r<p$ that is a very weak solution to (1.1), we can set $\epsilon=r-p(-1<\epsilon<0)$. Then there exists $\phi \in W_{0}^{1, \frac{r}{1+r-p}}(\Omega)$; thus, $\phi$ can be illustrated as a test-function in (1.2). In view of (2.1) and (2.2), we also can get an estimate of $D \phi$, which is similar to (2.2).

Applying Lemma 2, we can decompose the left term of the Hodge decomposition into two terms that could be controlled more easily in the proof of Theorem 1 .

Lemma 2 ([12]) For every $X, Y \in R^{n}, X \neq 0, Y \neq 0$, and $0 \leq \epsilon<1$, we have the inequality

$$
\left.|| X\right|^{-\epsilon} X-|Y|^{-\epsilon} Y\left|\leq 2^{\epsilon} \frac{1+\epsilon}{1-\epsilon}\right| X-\left.Y\right|^{1-\epsilon} .
$$

In the end of this section, we shall introduce a form of Gehring's lemma, which plays an important role in the proof of Theorem 1. It implies in particular that from it higher integrability of $g(x)$ follows.

Lemma 3 ([2,13]) Let $0<R<R_{0} \leq \operatorname{dist}\left(x_{0}, \partial \Omega\right), x_{0} \in \Omega$. Suppose that $g(x) \in L^{p}\left(B_{R}\left(x_{0}\right)\right)$, $f(x) \in L^{t}\left(B_{R}\left(x_{0}\right)\right), t>p, 1<p<\infty$, satisfy the reverse Hölder inequality

$$
f_{B_{R / 2}\left(x_{0}\right)}|g(x)|^{p} d x \leq \theta f_{B_{R}\left(x_{0}\right)}|g(x)|^{p} d x+C^{*}\left[f_{B_{R}\left(x_{0}\right)}|g(x)|^{s} d x\right]^{p / s}+f_{B_{R}\left(x_{0}\right)}|f(x)|^{p} d x
$$

for some $1 \leq s<p, 0 \leq \theta<1$. Then $g \in L_{\mathrm{loc}}^{p^{\prime}}(\Omega)$ for some $p^{\prime}=p^{\prime}\left(\theta, p, n, C^{*}\right)\left(t \geq p^{\prime}>p\right)$, and

$$
\left[f_{B_{R / 2}\left(x_{0}\right)}|g(x)|^{p^{\prime}} d x\right]^{1 / p^{\prime}} \leq C_{*}\left[f_{B_{R}\left(x_{0}\right)}|g(x)|^{p} d x\right]^{1 / p}+C_{*}\left[f_{B_{R}\left(x_{0}\right)}|f(x)|^{p^{\prime}} d x\right]^{1 / p^{\prime}},
$$

where $C_{*}=C_{*}\left(n, C^{*}, p, \theta, R_{0}\right)$.

\section{Proof of the main theorems}

In this section, we give a proof of partial regularity results. Consider $u$ solving (1.1) on $B_{R}\left(x_{0}\right) \Subset \Omega$, where we restrict $0<R<R_{0}<\min \left\{1\right.$, dist $\left.\left(x_{0}, \partial \Omega\right)\right\}$. 


\subsection{Proof of Theorem 1}

Proof Fix a cut-off function $\eta \in C_{0}^{\infty}\left(B_{R}\left(x_{0}\right)\right)$ satisfying $0 \leq \eta \leq 1,|D \eta| \leq C / R$, and $\eta \equiv 1$ on $B_{R / 2}\left(x_{0}\right)$. Let $u \in W_{\text {loc }}^{1, r}\left(B_{R}\left(x_{0}\right)\right)$ with $p-1<r<p$ be a very weak solution to (1.1). Denote $u-u_{x_{0}, R}-p_{0}\left(x-x_{0}\right)$ by $v$, where $p_{0} \in R^{n N}$. We find that $v$ has calculus mean-value 0 on $B_{R}\left(x_{0}\right)$, that is, $v_{x_{0}, R}=0$. Notice that $\eta v \in W_{0}^{1, r}\left(B_{R}\left(x_{0}\right)\right)$ and $-1<r-p<0$. Then there exist $\phi \in W_{0}^{1, \frac{r}{1+r-p}}\left(B_{R}\left(x_{0}\right)\right)$ and $h \in L^{\frac{r}{1+r-p}}\left(B_{R}\left(x_{0}\right)\right)$ such that $|D(\eta v)|^{r-p} D(\eta v)=D \phi+h$ according to the Hodge decomposition. Thus, $\phi$ is admissible as a test-function in the definition of very weak solutions. Set $-\varepsilon=r-p(-1<-\varepsilon<0)$ for convenience. Then $r=p-\varepsilon$, and we have

$$
|D(\eta v)|^{-\varepsilon} D(\eta v)=D \phi+h,
$$

where $h$ satisfies

$$
\|h\|_{\frac{p-\varepsilon}{1-\varepsilon}} \leq C_{r}(\Omega, N) \varepsilon\|D(\eta v)\|_{p-\varepsilon}^{1-\varepsilon} \text {. }
$$

Further, applying Poincaré's inequality with constant $C_{P}$ and noting that $v_{x_{0}, R}=0$, we get

$$
\begin{aligned}
\|D(\eta v)\|_{p-\varepsilon}^{1-\varepsilon} & \leq\left(\|v D \eta\|_{p-\varepsilon}+\|\eta D v\|_{p-\varepsilon}\right)^{1-\varepsilon} \\
& \leq\left(\frac{C}{R}\|v\|_{p-\varepsilon}+\|D v\|_{p-\varepsilon}\right)^{1-\varepsilon} \\
& \leq\left(C C_{P}^{\frac{1}{p-\varepsilon}}\|D v\|_{p-\varepsilon}+\|D v\|_{p-\varepsilon}\right)^{1-\varepsilon} \\
& <\left(1+C C_{P}^{\frac{1}{p-\varepsilon}}\right)\|D v\|_{p-\varepsilon}^{1-\varepsilon} .
\end{aligned}
$$

In view of (3.2) and (3.3), we have

$$
\|h\|_{\frac{p-\varepsilon}{1-\varepsilon}} \leq C_{1} \varepsilon\|D v\|_{p-\varepsilon}^{1-\varepsilon}
$$

where $C_{1}=C_{r}(\Omega, N)\left(1+C C_{P}^{\frac{1}{p-\varepsilon}}\right)$.

In particular, combining (3.1) and (3.2), we find

$$
\begin{aligned}
\|D \phi\|_{\frac{p-\varepsilon}{1-\varepsilon}} & =\left\||D(\eta v)|^{-\varepsilon} D(\eta v)-h\right\|_{\frac{p-\varepsilon}{1-\varepsilon}} \\
& \leq\left\||D(\eta v)|^{-\varepsilon} D(\eta v)\right\|_{\frac{p-\varepsilon}{1-\varepsilon}}+\|h\|_{\frac{p-\varepsilon}{1-\varepsilon}} \\
& \leq\|D(\eta v)\|_{p-\varepsilon}^{1-\varepsilon}+C_{r}(\Omega, N) \varepsilon\|D(\eta v)\|_{p-\varepsilon}^{1-\varepsilon} \\
& \leq\left(1+C_{r}(\Omega, N) \varepsilon\right)\|D(\eta v)\|_{p-\varepsilon}^{1-\varepsilon} .
\end{aligned}
$$

Substituting (3.3) into this estimate, we have

$$
\|D \phi\| \frac{p-\varepsilon}{1-\varepsilon} \leq C_{2}\|D \nu\|_{p-\varepsilon}^{1-\varepsilon},
$$

where $C_{2}=\left(1+C_{r}(\Omega, N) \varepsilon\right)\left(1+C C_{P}^{\frac{1}{p-\varepsilon}}\right)$. 
Since it is hard to estimate $|D(\eta v)|^{-\varepsilon} D(\eta v)$ directly, we set

$$
E(\eta, v)=|D(\eta v)|^{-\varepsilon} D(\eta v)-|\eta D v|^{-\varepsilon} \eta D v,
$$

which by Lemma 2 yields

$$
|E(\eta, v)| \leq 2^{\varepsilon} \frac{1+\varepsilon}{1-\varepsilon}|v D \eta|^{1-\varepsilon} .
$$

Joining $E(\eta, v)$ with (3.1), we arrive at

$$
D \phi=E(\eta, v)+|\eta D v|^{-\varepsilon} \eta D v-h .
$$

Inserting $D \phi$ into equality (1.2), we get

$$
\begin{aligned}
& \int_{B_{R}\left(x_{0}\right)} A(x, u, D u) \cdot|\eta D v|^{-\varepsilon} \eta D v d x \\
& \quad=\int_{B_{R}\left(x_{0}\right)} A(x, u, D u) \cdot h d x-\int_{B_{R}\left(x_{0}\right)} A(x, u, D u) \cdot E(\eta, v) d x+\int_{B_{R}\left(x_{0}\right)} f(x) \phi d x .
\end{aligned}
$$

In order to use (H2), we need to transform the left-hand side of (3.6) as follows:

$$
\begin{aligned}
& \int_{B_{R}\left(x_{0}\right)} A(x, u, D u) \cdot|\eta D v|^{-\varepsilon} \eta D v d x \\
& =\int_{B_{R}\left(x_{0}\right)}\left(A(x, u, D u)-A\left(x, u, p_{0}\right)+A\left(x, u, p_{0}\right)\right) \cdot|\eta D v|^{-\varepsilon} \eta D v d x \\
& =\int_{B_{R}\left(x_{0}\right)}\left(A(x, u, D u)-A\left(x, u, p_{0}\right)\right) \cdot|\eta D v|^{-\varepsilon} \eta D v d x \\
& \quad+\int_{B_{R}\left(x_{0}\right)} A\left(x, u, p_{0}\right) \cdot|\eta D v|^{-\varepsilon} \eta D v d x
\end{aligned}
$$

Combining this equality with (3.6), we find

$$
\begin{aligned}
\int_{B_{R}\left(x_{0}\right)} & \left(A(x, u, D u)-A\left(x, u, p_{0}\right)\right) \cdot|\eta D v|^{-\varepsilon} \eta D v d x \\
= & -\int_{B_{R}\left(x_{0}\right)} A\left(x, u, p_{0}\right) \cdot|\eta D v|^{-\varepsilon} \eta D v d x+\int_{B_{R}\left(x_{0}\right)} A(x, u, D u) \cdot h d x \\
\quad & -\int_{B_{R}\left(x_{0}\right)} A(x, u, D u) \cdot E(\eta, v) d x+\int_{B_{R}\left(x_{0}\right)} f(x) \phi d x \\
\leq & I_{1}+I_{2}+I_{3}+I_{4},
\end{aligned}
$$

where

$$
\begin{aligned}
& I_{1}=\left.\left|-\int_{B_{R}\left(x_{0}\right)} A\left(x, u, p_{0}\right) \cdot\right| \eta D v\right|^{-\varepsilon} \eta D v d x \mid ; \\
& I_{2}=\left|\int_{B_{R}\left(x_{0}\right)} A(x, u, D u) \cdot h d x\right|
\end{aligned}
$$




$$
\begin{aligned}
& I_{3}=\left|-\int_{B_{R}\left(x_{0}\right)} A(x, u, D u) \cdot E(\eta, v) d x\right| ; \\
& I_{4}=\left|\int_{B_{R}\left(x_{0}\right)} f(x) \phi d x\right| .
\end{aligned}
$$

Consequently, we shall derive estimate for each term of (3.7) so as to establish a reverse Hölder inequality for $\left|D u-p_{0}\right|^{p-\varepsilon}$.

In the case of the term on the left-hand side of (3.7), we want to derive an estimate from below in terms of $\int_{B_{R / 2}\left(x_{0}\right)}\left|D u-p_{0}\right|^{p-\varepsilon} d x$. For this purpose, we need the inequality

$$
(A(x, u, \zeta)-A(x, u, \xi)) \cdot(\zeta-\xi) \geq \alpha\left(1+|\zeta|^{2}+|\xi|^{2}\right)^{\frac{p-2}{2}}|\zeta-\xi|^{2}
$$

which can be deduced from (H2) immediately.

Then we infer that

$$
\begin{aligned}
& \int_{B_{R}\left(x_{0}\right)}\left(A(x, u, D u)-A\left(x, u, p_{0}\right)\right) \cdot|\eta D v|^{-\varepsilon} \eta D v d x \\
& \quad=\int_{B_{R}\left(x_{0}\right)}|\eta D v|^{-\varepsilon} \eta\left(A(x, u, D u)-A\left(x, u, p_{0}\right)\right) \cdot\left(D u-p_{0}\right) d x \\
& \quad \geq \alpha \int_{B_{R}\left(x_{0}\right)}|\eta D v|^{-\varepsilon} \eta\left(1+|D u|^{2}+\left|p_{0}\right|^{2}\right)^{\frac{p-2}{2}}\left|D u-p_{0}\right|^{2} d x \\
& \quad \geq \alpha \int_{B_{R / 2}\left(x_{0}\right)}\left|D u-p_{0}\right|^{2-\varepsilon}\left(1+\frac{\left|D u-p_{0}\right|^{2}}{2}\right)^{\frac{p-2}{2}} d x \\
& \quad \geq 2^{\frac{2-p}{2}} \alpha \int_{B_{R / 2}\left(x_{0}\right)}\left|D u-p_{0}\right|^{p-\varepsilon} d x .
\end{aligned}
$$

Using (H1) and Young's inequality with exponents $\frac{p-\varepsilon}{1-\varepsilon}$ and $\frac{p-\varepsilon}{p-1}$, we find that, for $\varepsilon_{1}>0$,

$$
\begin{aligned}
I_{1} \leq & \int_{B_{R}\left(x_{0}\right)}\left|A\left(x, u, p_{0}\right)\right||D v|^{1-\varepsilon} d x \\
\leq & \beta\left(1+\left|p_{0}\right|^{2}\right)^{\frac{p-1}{2}} \int_{B_{R}\left(x_{0}\right)}\left|D u-p_{0}\right|^{1-\varepsilon} d x \\
\leq & \beta\left(1+\left|p_{0}\right|^{2}\right)^{\frac{p-1}{2}} \int_{B_{R}\left(x_{0}\right)}\left(\varepsilon_{1}\left|D u-p_{0}\right|^{p-\varepsilon}+\varepsilon_{1}^{-\frac{1-\varepsilon}{p-1}} 1^{\frac{p-\varepsilon}{p-1}}\right) d x \\
\leq & \beta\left(1+\left|p_{0}\right|^{2}\right)^{\frac{p-1}{2}} \varepsilon_{1} \int_{B_{R}\left(x_{0}\right)}\left|D u-p_{0}\right|^{p-\varepsilon} d x \\
& +\beta\left(1+\left|p_{0}\right|^{2}\right)^{\frac{p-1}{2}} \varepsilon_{1}^{-\frac{1-\varepsilon}{p-1}} \int_{B_{R}\left(x_{0}\right)} d x .
\end{aligned}
$$

By (H1) we have

$$
\begin{aligned}
I_{2} & \leq \int_{B_{R}\left(x_{0}\right)}|A(x, u, D u)||h| d x \\
& \leq \beta \int_{B_{R}\left(x_{0}\right)}\left(1+|D u|^{2}\right)^{\frac{p-1}{2}}|h| d x
\end{aligned}
$$


Zhao and Chen Journal of Inequalities and Applications （2017） 2017:23

Page 8 of 12

$$
\begin{aligned}
& \leq \beta \int_{B_{R}\left(x_{0}\right)}\left(2+\left|D u-p_{0}+p_{0}\right|^{2}\right)^{\frac{p-1}{2}}|h| d x \\
& \leq \beta \int_{B_{R}\left(x_{0}\right)}\left(2\left(1+\left|p_{0}\right|^{2}\right)+2\left|D u-p_{0}\right|^{2}\right)^{\frac{p-1}{2}}|h| d x \\
& \leq 2^{p-1} \beta \int_{B_{R}\left(x_{0}\right)}\left|D u-p_{0}\right|^{p-1}|h| d x+2^{p-1} \beta\left(1+\left|p_{0}\right|^{2}\right)^{\frac{p-1}{2}} \int_{B_{R}\left(x_{0}\right)}|h| d x .
\end{aligned}
$$

Applying both Hölder's inequality and Young's inequality with exponents $\frac{p-\varepsilon}{p-1}$ and $\frac{p-\varepsilon}{1-\varepsilon}$, by (3.4) we further have that, for $\varepsilon_{2}>0$,

$$
\begin{aligned}
I_{2} \leq & 2^{p-1} \beta\left(\int_{B_{R}\left(x_{0}\right)}\left|D u-p_{0}\right|^{p-\varepsilon} d x\right)^{\frac{p-1}{p-\varepsilon}}\left(\int_{B_{R}\left(x_{0}\right)}|h|^{\frac{p-\varepsilon}{1-\varepsilon}} d x\right)^{\frac{1-\varepsilon}{p-\varepsilon}} \\
& +2^{p-1} \beta\left(1+\left|p_{0}\right|^{2}\right)^{\frac{p-1}{2}}\left(\int_{B_{R}\left(x_{0}\right)} 1^{p-\varepsilon} d x\right)^{\frac{p-1}{p-\varepsilon}}\left(\int_{B_{R}\left(x_{0}\right)}|h|^{\frac{p-\varepsilon}{1-\varepsilon}} d x\right)^{\frac{1-\varepsilon}{p-\varepsilon}} \\
\leq & 2^{p-1} \beta C_{1} \varepsilon\left(\int_{B_{R}\left(x_{0}\right)}\left|D u-p_{0}\right|^{p-\varepsilon} d x\right)^{\frac{p-1}{p-\varepsilon}}\left(\int_{B_{R}\left(x_{0}\right)}\left|D u-p_{0}\right|^{p-\varepsilon} d x\right)^{\frac{1-\varepsilon}{p-\varepsilon}} \\
& +2^{p-1} \beta\left(1+\left|p_{0}\right|^{2}\right)^{\frac{p-1}{2}} C_{1} \varepsilon\left(\int_{B_{R}\left(x_{0}\right)}\left|D u-p_{0}\right|^{p-\varepsilon} d x\right)^{\frac{1-\varepsilon}{p-\varepsilon}}\left(\int_{B_{R}\left(x_{0}\right)} d x\right)^{\frac{p-1}{p-\varepsilon}} \\
\leq & 2^{p-1} \beta C_{1} \varepsilon \int_{B_{R}\left(x_{0}\right)}\left|D u-p_{0}\right|^{p-\varepsilon} d x \\
& +2^{p-1} \beta\left(1+\left|p_{0}\right|^{2}\right)^{\frac{p-1}{2}} C_{1} \varepsilon\left(\varepsilon_{2} \int_{B_{R}\left(x_{0}\right)}\left|D u-p_{0}\right|^{p-\varepsilon} d x+\varepsilon_{2}^{-\frac{1-\varepsilon}{p-1}} \int_{B_{R}\left(x_{0}\right)} d x\right) \\
\leq & 2^{p-1} \beta C_{1} \varepsilon \int_{B_{R}\left(x_{0}\right)}\left|D u-p_{0}\right|^{p-\varepsilon} d x+2^{p-1} \beta\left(1+\left|p_{0}\right|^{2}\right)^{\frac{p-1}{2}} C_{1} \varepsilon \varepsilon_{2}^{-\frac{1-\varepsilon}{p-1}} \int_{B_{R}\left(x_{0}\right)} d x \\
& +2^{p-1} \beta\left(1+\left|p_{0}\right|^{2}\right)^{\frac{p-1}{2}} C_{1} \varepsilon \varepsilon_{2} \int_{B_{R}\left(x_{0}\right)}^{\left|D u-p_{0}\right|^{p-\varepsilon} d x .}
\end{aligned}
$$

Combining (H1) and the estimate of $E(\eta, v)$, we find that

$$
\begin{aligned}
I_{3} & \leq \int_{B_{R}\left(x_{0}\right)}|A(x, u, D u)||E(\eta, v)| d x \\
& \leq \int_{B_{R}\left(x_{0}\right)} \beta\left(1+|D u|^{2}\right)^{\frac{p-1}{2}} 2^{\varepsilon} \frac{1+\varepsilon}{1-\varepsilon}|v D \eta|^{1-\varepsilon} d x \\
& \leq \beta 2^{\varepsilon} \frac{1+\varepsilon}{1-\varepsilon}\left(\frac{C}{R}\right)^{1-\varepsilon} \int_{B_{R}\left(x_{0}\right)}\left(1+|D u|^{2}\right)^{\frac{p-1}{2}}|v|^{1-\varepsilon} d x . \\
& \leq \beta 2^{\varepsilon} \frac{1+\varepsilon}{1-\varepsilon}\left(\frac{C}{R}\right)^{1-\varepsilon} \int_{B_{R}\left(x_{0}\right)}\left(2\left(1+\left|p_{0}\right|^{2}\right)+2\left|D u-p_{0}\right|^{2}\right)^{\frac{p-1}{2}}|v|^{1-\varepsilon} d x \\
& \leq \beta 2^{\varepsilon} \frac{1+\varepsilon}{1-\varepsilon}\left(\frac{C}{R}\right)^{1-\varepsilon} 2^{p-1} \int_{B_{R}\left(x_{0}\right)}\left(\left(1+\left|p_{0}\right|^{2}\right)^{\frac{p-1}{2}}+\left|D u-p_{0}\right|^{p-1}\right)|v|^{1-\varepsilon} d x .
\end{aligned}
$$

Denoting $\beta 2^{\varepsilon} \frac{1+\varepsilon}{1-\varepsilon}\left(\frac{C}{R}\right)^{1-\varepsilon} 2^{p-1}$ by $C_{3}$, we have

$$
I_{3} \leq C_{3}\left(1+\left|p_{0}\right|^{2}\right)^{\frac{p-1}{2}} K_{1}+C_{3} K_{2},
$$


where $K_{1}=\int_{B_{R}\left(x_{0}\right)}|v|^{1-\varepsilon} d x$ and $K_{2}=\int_{B_{R}\left(x_{0}\right)}\left|D u-p_{0}\right|^{p-1}|v|^{1-\varepsilon} d x$. Let us estimate $K_{1}$ and $K_{2}$. Using Young's inequality with exponents $\frac{p-\varepsilon}{1-\varepsilon}$ and $\frac{p-\varepsilon}{p-1}$ and Poincaré's inequality with constant $C_{P}$, we find that, for $\varepsilon_{3}>0$,

$$
\begin{aligned}
K_{1} & \leq \int_{B_{R}\left(x_{0}\right)}\left(\varepsilon_{3}|v|^{p-\varepsilon}+\varepsilon_{3}^{-\frac{1-\varepsilon}{p-1}} 1^{\frac{p-\varepsilon}{p-1}}\right) d x \\
& \leq \varepsilon_{3} \int_{B_{R}\left(x_{0}\right)}|v|^{p-\varepsilon} d x+\varepsilon_{3}^{-\frac{1-\varepsilon}{p-1}} \int_{B_{R}\left(x_{0}\right)} d x \\
& \leq \varepsilon_{3} C_{P} \int_{B_{R}\left(x_{0}\right)}\left|D u-p_{0}\right|^{p-\varepsilon} d x+\varepsilon_{3}^{-\frac{1-\varepsilon}{p-1}} \int_{B_{R}\left(x_{0}\right)} d x .
\end{aligned}
$$

Letting $p^{\prime}=\frac{n(p-\varepsilon)}{(n+1-\varepsilon)(p-1)}$ and $q^{\prime}=\frac{n(p-\varepsilon)}{(n-p+1)(1-\varepsilon)}$, we see that $1<p^{\prime}<\infty, 1<q^{\prime}<\infty$, and $\frac{1}{p^{\prime}}+\frac{1}{q^{\prime}}=1$. With the aid of Hölder's inequality, we can estimate

$$
\begin{aligned}
K_{2} & \leq\left(\int_{B_{R}\left(x_{0}\right)}\left|D u-p_{0}\right|^{(p-1) p^{\prime}} d x\right)^{\frac{1}{p^{\prime}}}\left(\int_{B_{R}\left(x_{0}\right)}|\nu|^{(1-\varepsilon) q^{\prime}} d x\right)^{\frac{1}{q^{\prime}}} \\
& \leq\left(\int_{B_{R}\left(x_{0}\right)}\left|D u-p_{0}\right|^{\frac{n(p-\varepsilon)}{n+1-\varepsilon}} d x\right)^{\frac{(n+1-\varepsilon)(p-1)}{n(p-\varepsilon)}}\left(\int_{B_{R}\left(x_{0}\right)}|v|^{\frac{n(p-\varepsilon)}{n-p+1}} d x\right)^{\frac{(n-p+1)(1-\varepsilon)}{n(p-\varepsilon)}} .
\end{aligned}
$$

Now we set $p^{\prime \prime}=\frac{n(p-\varepsilon)}{n+1-\varepsilon}$. Then $\frac{n p^{\prime \prime}}{n-p^{\prime \prime}}=\frac{n(p-\varepsilon)}{n-p+1}$. Using the Sobolev-Poincaré inequality with constant $C_{s}$, we get

$$
\begin{aligned}
K_{2} & \leq C_{s}^{1-\varepsilon}\left(\int_{B_{R}\left(x_{0}\right)}\left|D u-p_{0}\right|^{\frac{n(p-\varepsilon)}{n+1-\varepsilon}} d x\right)^{\frac{(n+1-\varepsilon)(p-1)}{n(p-\varepsilon)}}\left(\int_{B_{R}\left(x_{0}\right)}\left|D u-p_{0}\right|^{\frac{n(p-\varepsilon)}{n+1-\varepsilon}} d x\right)^{\frac{(n+1-\varepsilon)(1-\varepsilon)}{n(p-\varepsilon)}} \\
& \leq C_{s}^{1-\varepsilon}\left(\int_{B_{R}\left(x_{0}\right)}\left|D u-p_{0}\right|^{\frac{n(p-\varepsilon)}{n+1-\varepsilon}} d x\right)^{\frac{n+1-\varepsilon}{n}}
\end{aligned}
$$

Combining (3.11) with (3.12) and (3.13), we obtain the estimate for $I_{3}$ :

$$
\begin{aligned}
I_{3} \leq & C_{3}\left(1+\left|p_{0}\right|^{2}\right)^{\frac{p-1}{2}} \varepsilon_{3} C_{P} \int_{B_{R}\left(x_{0}\right)}\left|D u-p_{0}\right|^{p-\varepsilon} d x \\
& +C_{3}\left(1+\left|p_{0}\right|^{2}\right)^{\frac{p-1}{2}} \varepsilon_{3}^{-\frac{1-\varepsilon}{p-1}} \int_{B_{R}\left(x_{0}\right)} d x \\
& +C_{3} C_{s}^{1-\varepsilon}\left(\int_{B_{R}\left(x_{0}\right)}\left|D u-p_{0}\right|^{\frac{n(p-\varepsilon)}{n+1-\varepsilon}} d x\right)^{\frac{n+1-\varepsilon}{n}} .
\end{aligned}
$$

Finally, we estimate $I_{4}$. Using Hölder's inequality with exponents $\frac{n(p-\varepsilon)}{n(p-1)+p-\varepsilon}$ and $\frac{n(p-\varepsilon)}{n(1-\varepsilon)-p+\varepsilon}$, we have

$$
\begin{aligned}
I_{4} & \leq \int_{B_{R}\left(x_{0}\right)}|f(x)||\phi| d x \\
& \leq\left(\int_{B_{R}\left(x_{0}\right)}|f|^{\frac{n(p-\varepsilon)}{n(p-1)+p-\varepsilon}} d x\right)^{\frac{n(p-1)+p-\varepsilon}{n(p-\varepsilon)}}\left(\int_{B_{R}\left(x_{0}\right)}|\phi|^{\frac{n(p-\varepsilon)}{n(1-\varepsilon)-p+\varepsilon}} d x\right)^{\frac{n(1-\varepsilon)-p+\varepsilon}{n(p-\varepsilon)}} .
\end{aligned}
$$


Zhao and Chen Journal of Inequalities and Applications ( 2017) 2017:23

Page 10 of 12

Setting $p^{\prime \prime \prime}=\frac{p-\varepsilon}{1-\varepsilon}$, we have $\frac{n p^{\prime \prime \prime}}{n-p^{\prime \prime \prime}}=\frac{n(p-\varepsilon)}{n(1-\varepsilon)-p+\varepsilon}$. Notice that $\phi \in W_{0}^{1, \frac{p-\varepsilon}{1-\varepsilon}}\left(B_{R}\left(x_{0}\right)\right)$. Therefore, we can apply the Sobolev-Poincaré inequality to get

$$
I_{4} \leq C_{s}\left(\int_{B_{R}\left(x_{0}\right)}|f|^{\frac{n(p-\varepsilon)}{n(p-1)+p-\varepsilon}} d x\right)^{\frac{n(p-1)+p-\varepsilon}{n(p-\varepsilon)}}\left(\int_{B_{R}\left(x_{0}\right)}|D \phi|^{\frac{p-\varepsilon}{1-\varepsilon}} d x\right)^{\frac{1-\varepsilon}{p-\varepsilon}}
$$

Combining this with (3.5), with the aid of Young's inequality, we obtain, for $\varepsilon_{4}>0$,

$$
\begin{aligned}
I_{4} & \leq C_{s} C_{2}\left(\int_{B_{R}\left(x_{0}\right)}|f|^{\frac{n(p-\varepsilon)}{n(p-1)+p-\varepsilon}} d x\right)^{\frac{n(p-1)+p-\varepsilon}{n(p-\varepsilon)}}\left(\int_{B_{R}\left(x_{0}\right)}\left|D u-p_{0}\right|^{p-\varepsilon} d x\right)^{\frac{1-\varepsilon}{p-\varepsilon}} \\
& \leq C_{s} C_{2}\left(\varepsilon_{4} \int_{B_{R}\left(x_{0}\right)}\left|D u-p_{0}\right|^{p-\varepsilon} d x+\varepsilon_{4}^{-\frac{1-\varepsilon}{p-1}}\left(\int_{B_{R}\left(x_{0}\right)}|f|^{\frac{n(p-\varepsilon)}{n(p-1)+p-\varepsilon}} d x\right)^{\frac{n(p-1)+p-\varepsilon}{n(p-1)}}\right) .
\end{aligned}
$$

Substituting (3.8), (3.9), (3.10), (3.14), and (3.15) into (3.7), we finally have

$$
\begin{aligned}
2^{\frac{2-p}{2}} \alpha & \int_{B_{R / 2}\left(x_{0}\right)}\left|D u-p_{0}\right|^{p-\varepsilon} d x \\
\leq & \beta\left(1+\left|p_{0}\right|^{2}\right)^{\frac{p-1}{2}} \varepsilon_{1} \int_{B_{R}\left(x_{0}\right)}\left|D u-p_{0}\right|^{p-\varepsilon} d x+2^{p-1} \beta C_{1} \varepsilon \int_{B_{R}\left(x_{0}\right)}\left|D u-p_{0}\right|^{p-\varepsilon} d x \\
& +2^{p-1} \beta\left(1+\left|p_{0}\right|^{2}\right)^{\frac{p-1}{2}} C_{1} \varepsilon \varepsilon_{2} \int_{B_{R}\left(x_{0}\right)}\left|D u-p_{0}\right|^{p-\varepsilon} d x \\
& +C_{3}\left(1+\left|p_{0}\right|^{2}\right)^{\frac{p-1}{2}} \varepsilon_{3} C_{P} \int_{B_{R}\left(x_{0}\right)}\left|D u-p_{0}\right|^{p-\varepsilon} d x+C_{s} C_{2} \varepsilon_{4} \int_{B_{R}\left(x_{0}\right)}\left|D u-p_{0}\right|^{p-\varepsilon} d x \\
& +C_{3} C_{s}^{1-\varepsilon}\left(\int_{B_{R}\left(x_{0}\right)}\left|D u-p_{0}\right|^{\frac{n(p-\varepsilon)}{n+1-\varepsilon}} d x\right)^{\frac{n+1-\varepsilon}{n}} \\
& +C_{s} C_{2} \varepsilon_{4}^{-\frac{1-\varepsilon}{p-1}}\left(\int_{B_{R}\left(x_{0}\right)}|f|^{\frac{n(p-\varepsilon)}{n(p-1)+p-\varepsilon}} d x\right)^{\frac{n(p-1)+p-\varepsilon}{n(p-1)}} \\
& +\beta\left(1+\left|p_{0}\right|^{2}\right)^{\frac{p-1}{2}} \varepsilon_{1}^{-\frac{1-\varepsilon}{p-1}} \int_{B_{R}\left(x_{0}\right)} d x+2^{p-1} \beta\left(1+\left|p_{0}\right|^{2}\right)^{\frac{p-1}{2}} C_{1} \varepsilon \varepsilon_{2}^{-\frac{1-\varepsilon}{p-1}} \int_{B_{R}\left(x_{0}\right)} d x \\
& +C_{3}\left(1+\left|p_{0}\right|^{2}\right)^{\frac{p-1}{2}} \varepsilon_{3}^{-\frac{1-\varepsilon}{p-1}} \int_{B_{R}\left(x_{0}\right)} d x .
\end{aligned}
$$

Rearranging this inequality, we have

$$
\begin{gathered}
\int_{B_{R / 2}\left(x_{0}\right)}\left|D u-p_{0}\right|^{p-\varepsilon} d x \\
\leq \theta \int_{B_{R}\left(x_{0}\right)}\left|D u-p_{0}\right|^{p-\varepsilon} d x+\frac{1}{\alpha} 2^{\frac{p-2}{2}} C_{3} C_{s}^{1-\varepsilon}\left(\int_{B_{R}\left(x_{0}\right)}\left|D u-p_{0}\right|^{\tau} d x\right)^{\frac{p-\varepsilon}{\tau}} \\
\quad+\frac{1}{\alpha} 2^{\frac{p-2}{2}} C_{s} C_{2} \varepsilon_{4}^{-\frac{1-\varepsilon}{p-1}}\left(\int_{B_{R}\left(x_{0}\right)}|f|^{\frac{n(p-\varepsilon)}{n(p-1)+p-\varepsilon}} d x\right)^{\frac{n(p-1)+p-\varepsilon}{n(p-1)}}+C_{4} \int_{B_{R}\left(x_{0}\right)} d x, \\
\text { where } \theta=\frac{1}{\alpha} 2^{\frac{p-2}{2}}\left(\beta\left(1+\left|p_{0}\right|^{2}\right)^{\frac{p-1}{2}} \varepsilon_{1}+2^{p-1} \beta C_{1} \varepsilon+2^{p-1} \beta\left(1+\left|p_{0}\right|^{2}\right)^{\frac{p-1}{2}} C_{1} \varepsilon \varepsilon_{2}+C_{3}\left(1+\left|p_{0}\right|^{2}\right)^{\frac{p-1}{2}} \times\right. \\
\left.\varepsilon_{3} C_{P}+C_{s} C_{2} \varepsilon_{4}\right), \tau=\frac{n(p-\varepsilon)}{n+1-\varepsilon}, \text { and } C_{4}=\frac{1}{\alpha} 2^{\frac{p-2}{2}}\left(\beta\left(1+\left|p_{0}\right|^{2}\right)^{\frac{p-1}{2}} \varepsilon_{1}^{-\frac{1-\varepsilon}{p-1}}+2^{p-1} \beta\left(1+\left|p_{0}\right|^{2}\right)^{\frac{p-1}{2}} C_{1} \varepsilon \times\right. \\
\left.\varepsilon_{2}^{-\frac{1-\varepsilon}{p-1}}+C_{3}\left(1+\left|p_{0}\right|^{2}\right)^{\frac{p-1}{2}} \varepsilon_{3}^{-\frac{1-\varepsilon}{p-1}}\right) .
\end{gathered}
$$


By (H4) we get $\frac{n(p-\varepsilon)}{n(p-1)+p-\varepsilon}<\frac{n q}{n(p-1)+q}$ and $\int_{B_{R}\left(x_{0}\right)}|f|^{\frac{n(p-\varepsilon)}{n(p-1)+p-\varepsilon}} d x<M$.

Moreover, we have $\left(\int_{B_{R}\left(x_{0}\right)}|f|^{\frac{n(p-\varepsilon)}{n(p-1)+p-\varepsilon}} d x\right)^{\frac{n(p-1)+p-\varepsilon}{n(p-1)}} \leq M^{\frac{p-\varepsilon}{n(p-1)}} \int_{B_{R}\left(x_{0}\right)}|f|^{\frac{n(p-\varepsilon)}{n(p-1)+p-\varepsilon}} d x$.

Setting $C_{5}=\max \left\{\frac{1}{\alpha} 2^{\frac{p-2}{2}} C_{s} C_{2} \varepsilon_{4}^{-\frac{1-\varepsilon}{p-1}} M^{\frac{p-\varepsilon}{n(p-1)}}, C_{4}\right\}$, we have

$$
\begin{aligned}
& \int_{B_{R / 2}\left(x_{0}\right)}\left|D u-p_{0}\right|^{p-\varepsilon} d x \\
& \leq \theta \int_{B_{R}\left(x_{0}\right)}\left|D u-p_{0}\right|^{p-\varepsilon} d x+\frac{1}{\alpha} 2^{\frac{p-2}{2}} C_{3} C_{s}^{1-\varepsilon}\left(\int_{B_{R}\left(x_{0}\right)}\left|D u-p_{0}\right|^{\tau} d x\right)^{\frac{p-\varepsilon}{\tau}} \\
& \quad+C_{5}\left(\int_{B_{R}\left(x_{0}\right)}|f|^{\frac{n}{n(p-1)+p-\varepsilon}(p-\varepsilon)} d x+\int_{B_{R}\left(x_{0}\right)} d x\right) \\
& \leq \\
& \quad \int_{B_{R}\left(x_{0}\right)}\left|D u-p_{0}\right|^{p-\varepsilon} d x+\frac{1}{\alpha} 2^{\frac{p-2}{2}} C_{3} C_{s}^{1-\varepsilon}\left(\int_{B_{R}\left(x_{0}\right)}\left|D u-p_{0}\right|^{\tau} d x\right)^{\frac{p-\varepsilon}{\tau}} \\
& \quad+C_{5} \int_{B_{R}\left(x_{0}\right)}\left(|f|^{\frac{n}{n(p-1)+p-\varepsilon}}+1\right)^{p-\varepsilon} d x .
\end{aligned}
$$

Dividing both sides by $\left|B_{R}\left(x_{0}\right)\right|=\alpha_{n} R^{n}$ yields

$$
\begin{aligned}
& f_{B_{R / 2}\left(x_{0}\right)}\left|D u-p_{0}\right|^{p-\varepsilon} d x \\
& \leq 2^{n} \theta-\int_{B_{R}\left(x_{0}\right)}\left|D u-p_{0}\right|^{p-\varepsilon} d x+C_{6}\left(f_{B_{R}\left(x_{0}\right)}\left|D u-p_{0}\right|^{\tau} d x\right)^{\frac{p-\varepsilon}{\tau}} \\
& \quad+2^{n} C_{5} f_{B_{R}\left(x_{0}\right)}\left(|f|^{\frac{n}{n(p-1)+p-\varepsilon}}+1\right)^{p-\varepsilon} d x
\end{aligned}
$$

where $C_{6}=\frac{2^{n}}{\alpha} 2^{\frac{p-2}{2}} C_{3} C_{s}^{1-\varepsilon}\left(\alpha_{n} R^{n}\right)^{\frac{p-\tau-\varepsilon}{\tau}}$.

Taking $\varepsilon, \varepsilon_{1}, \varepsilon_{2}, \varepsilon_{3}, \varepsilon_{4}$ sufficiently small such that $2^{n} \theta<1$ and $\tau>1$, we obtain the reverse Hölder inequality for $\left|D u-p_{0}\right|^{p-\varepsilon}$. Accordingly, by Lemma 2 we can derive that $u$ belongs to $W_{\text {loc }}^{1, r^{\prime}}(\Omega)$ with $r^{\prime}>r$. Since $f \in L_{\text {loc }}^{\frac{n q}{n(p-1)+q}}(\Omega), q>p$, reasoning as before, we get a new estimate analogous to (3.16) with exponents $r^{\prime}$ and $\tau^{\prime}$ in place of $r$, that is, $p-\varepsilon$ and $\tau$, respectively:

$$
\begin{aligned}
& f_{B_{R / 2}\left(x_{0}\right)}\left|D u-p_{0}\right|^{r^{\prime}} d x \\
& \leq 2^{n} \theta^{\prime} f_{B_{R}\left(x_{0}\right)}\left|D u-p_{0}\right|^{r^{\prime}} d x+C_{6}^{\prime}\left(f_{B_{R}\left(x_{0}\right)}\left|D u-p_{0}\right|^{\tau^{\prime}} d x\right)^{\frac{r^{\prime}}{\tau^{\prime}}} \\
& \quad+2^{n} C_{5}^{\prime} f_{B_{R}\left(x_{0}\right)}\left(|f|^{\frac{n}{n(p-1)+r^{\prime}}}+1\right)^{r^{\prime}} d x
\end{aligned}
$$

Therefore, we get $u \in W_{\text {loc }}^{1, r^{\prime \prime}}(\Omega)$ with $r^{\prime \prime}>r^{\prime}$. Repeating this process, we can improve the degree of integrability of $D u$ again and again. Thus, it is clear that $u \in W_{\text {loc }}^{1, t}(\Omega)$ with any $t \in\left(r_{1}, r_{2}\right)$.

This completes the proof of Theorem 1 . 


\subsection{Proof of Theorem 2}

Proof The aim of Theorem 2 is to prove that very weak solutions to systems (1.1) are not only weak solutions to (1.1) but also the optimal Hölder continuity. In fact, under the assumptions of Theorem 1, we get that $u \in W_{\text {loc }}^{1, r}(\Omega)$ with $r_{1}<r<p$ are weak solutions $u \in W_{\text {loc }}^{1, p}(\Omega)$ to systems (1.1) by Corollary 1 . Then we can safely infer $u \in C^{1, \gamma}\left(\Omega_{0}\right)$ based on A-harmonic approximation technique. The proving method is standard, so we omit the process of derivation in this paper. For more details, we refer the reader to Theorem 1.1 of [14] and the related literature. So the proof of Theorem 2 is complete.

Competing interests

The authors declare that they have no competing interests.

Authors' contributions

Both authors contributed equally to writing of this paper. Both authors read and approved the final manuscript.

\section{Acknowledgements}

The authors would like to thank the anonymous referee for careful reading the manuscript and valuable comments. This work was supported by the National Natural Science foundation of China under Grant No. 11571159.

Received: 26 November 2016 Accepted: 3 January 2017 Published online: 18 January 2017

\section{References}

1. Gehring, FW: The $L^{P}$-integrability of the partial derivatives of a quasiconformal mapping. Acta Math. 130, 265-277 (1973)

2. Meyers, N, Elcrat, A: Some results on regularity for solutions of non-linear elliptic systems and quasi-regular functions. Duke Math. J. 42(1), 121-136 (1975)

3. Meyers, $N$ : An $L^{P}$-estimate for the gradient of solutions of second order elliptic divergence equations. Ann. Sc. Norm. Super. Pisa, Cl. Sci. 17, 189-206 (1963)

4. Lewis, JL: On the very weak solutions of certain elliptic systems. Commun. Partial Differ. Equ. 18, 1515-1537 (1993)

5. Iwaniec, T, Sbordone, C: Weak minima of variational integrals. J. Reine Angew. Math. 454, 143-161 (1994)

6. Giachetti, D, Leonetti, F, Schiachi, R: On the regularity of very weak minimal. Proc. R. Soc. Edinb. A 126, $287-296$ (1996)

7. Tong, YX, Gu, JT, Xu, XJ: Regularity for very weak solutions to A-harmonic equation. Appl. Math. J. Chin. Univ. Ser. A 24(3), 319-323 (2009)

8. Greco, L, Iwaniec, T, Sbordone, C: Inverting the $p$-harmonic operator. Manuscr. Math. 92, 249-258 (1997)

9. De Giorgi, E: Frontiere orientate di misura minima. Seminaro Mat. Scuola Norm. Sup. Pisa. Editrice Tecnico Scientifica, Pisa (1961)

10. De Giorgi, E: Un esempio di estremali discontinue per un problema variazionale di tipo ellitico. Boll. Unione Mat. Ital. 4, 135-137 (1968)

11. Giusti, E, Miranda, M: Sulla regolarità delle soluzioni deboli di una classe di sistemi ellittici quasi-lineari. Arch. Ration. Mech. Anal. 31, 173-184 (1968)

12. Iwaniec, T, Migliaccio, L, Nania, L, Sbordone, C: Integrability and removability results for quasiregular mappings in high dimensions. Math. Scand. 75(2), 263-279 (1994)

13. Giaquinta, M: Multiple Integrals in the Calculus of Variations and Nonlinear Elliptic Systems. Princeton University Press, Princeton (1983)

14. Chen, SH, Tan, Z: Optimal interior partial regularity for nonlinear elliptic systems under the natural growth condition: the method of A-harmonic approximation. Acta Math. Sci. 27B(3), 491-508 (2007)

\section{Submit your manuscript to a SpringerOpen ${ }^{\circ}$ journal and benefit from:}

- Convenient online submission

Rigorous peer review

- Immediate publication on acceptance

- Open access: articles freely available online

- High visibility within the field

- Retaining the copyright to your article 